\title{
Exposições Científicas como Ferramenta de Obtenção de Dados para Desenvolvimento de Estratégias de Conservação de Quelônios Amazônicos
}

\section{Scientific Exhibitions as a Tool for Obtaining Data for the Development of Conservation Strategies for Amazonian Chelonians}

\author{
Matheus Nascimento Oliveiraa ; Isaac Ibernon Lopes-Filho ${ }^{\mathrm{b}}$; Victor Silva Vasconcelos ${ }^{\mathrm{b}}$; Marilene Vasconcelos da Silva \\ Brazil $^{c}$; Ester Nascimento da Costa ${ }^{a}$; Maria Isabel Afonso da Silva ${ }^{a}$; Tiago Lucena da Silva ${ }^{a}$ \\ ${ }^{a}$ Universidade Federal do Acre. AC, Brasil. \\ bUniversidade Federal do Acre, Programa de Pós-Graduação Stricto Sensu em Sanidade e Produção Animal Sustentável. AC, Brasil. \\ 'Universidade Federal do Acre, Programa de Pós-Graduação Stricto Sensu em Biodiversidade e Biotecnologia da Amazônia Legal. AC, Brasil. \\ *E-mail: oliveiramatheus.bio@gmail.com
}

\begin{abstract}
Resumo
As exposições científicas são ferramentas metodológicas importantes para promover conhecimentos de forma dinâmica e de fácil compreensão ao público participante. Com a intenção de proporcionar esses conhecimentos a um público maior e diversificado, foi desenvolvido pela equipe do Projeto Bichos de Casco vinculado ao Laboratório de Biologia Animal da Universidade Federal do Acre (Ufac) - Campus Floresta, uma exposição com base na conservação de quelônios amazônicos ocorrentes no Alto Juruá, para ser aplicada durante o evento regional Expo Acre Juruá, entre agosto e setembro de 2017, que contou com a presença de um público estimado de 20 mil pessoas por dia, segundo dados do SEBRAE (Serviço Brasileiro de Apoio às Micro e Pequenas Empresas). Durante a exposição, foram aplicados questionários semiestruturados com o intuito de fazer um levantamento de dados Etnozoológicos acerca de quelônios no perímetro urbano da cidade de Cruzeiro do Sul - Acre, registrando dados socioeconômicos e de consumo para assim possibilitar um melhor direcionamento para a realização de futuros trabalhos focados na conservação de quelônios na região. De acordo com os dados, 50\% dos entrevistados afirmaram utilizar quelônios na alimentação, sendo, em sua maioria, indivíduos do sexo masculino. A espécie mais relatada no consumo foi o Jabuti (Chelonoidis denticulatus), o que demostra que a espécie é muito apreciada na região. Com os resultados se pode concluir que exposições científicas são de grande valia para popularização da Ciência e obtenção de dados para subsidiar ações de conservação.
\end{abstract}

Palavras-chave: Recursos Naturais. Jabutis. Subsistência.

\begin{abstract}
Scientific exhibitions are important methodological tools to promote knowledge in a dynamic and easy way of understanding the participating public. With the intention of providing this knowledge to a larger and diversified public, it was developed by the Bichos de Casco Project team linked to the Animal Biology Laboratory of the Federal University of Acre (Ufac) - Campus Floresta, an exhibition based on the conservation of Amazonian chelonians occurring in Alto Juruá, to be applied during the regional ExpoAcre Juruá event between August and September 2017, which was attended by an estimated audience of 20 thousand people per day, according to data from SEBRAE (Brazilian Support Service for Micro and Small Companies). During the exhibition, semi-structured questionnaires were applied in order to survey Ethnozoological data about chelonians in the urban perimeter of Cruzeiro do Sul - Acre, recording socioeconomic and consumption data to enable a better direction for future works focused on the conservation of chelonians in the region. According to the data, half of the interviewees stated that they used chelonians as food resource, being mostly male individuals, the species most reported in consumption was the yellow-footed tortoise (Chelonoidis denticulatus), which shows that the species is very appreciated in the region. With the results, it can be concluded that scientific exhibitions are of great value for popularizing science and obtaining data to support conservation actions.
\end{abstract}

Keywords: Natural Resources. Tortoises; Subsistence.

\section{Introdução}

A caça e a pesca se apresentam como importantes práticas culturais na Região Amazônica, principalmente como fonte de aquisição alimentar para a subsistência de comunidades tradicionais e povos indígenas (TERRA, 2007). Entre os grupos mais consumidos por essas populações estão os quelônios, também conhecidos regionalmente como bichos de casco, grupo que engloba tartarugas, cágados e jabutis (MOLL; MOLL; RIEDLE, 2004), que continuam sendo utilizados, até os dias atuais, na culinária tradicional e na dieta de comunitários, ribeirinhos, extrativistas e povos indígenas (FACHIN-TERÁN, 2005; REBÊLO; OLIVEIRA et al., 2016; PEZZUTI, 2000; SILVA; BEGOSSI, 2003).
Eisemberg e Vogt (2015) afirmaram que o comércio ilegal é o principal fator que influencia na diminuição populacional de quelônios na Amazônia, seguido pela captura desses animais em seu período reprodutivo, além do longo histórico cultural de uso desses animais e seus subprodutos, como: ovos, carne, gorduras e vísceras. Diante do consumo exacerbado na Região Amazônica, os quelônios estão entre os animais que sofrem maior impacto na sua população natural (CANTARELLI; MALVASIO; VERDADE, 2014), entre eles o Tracajá (Podocnemis unifilis), Cabeçudo (Peltocephalus dumerilianus), Tartaruga da Amazônia (Podocnemis expansa), Irapuca (Podocnemis erythrocephala), Iaçá (Podocnemis sextuberculata) e as duas espécies de Jabutis, (Chelonoidis denticulatus e C. carbonarius) (REBÊLO; PEZZUTI, 2000; 


\section{VOGT, 2008).}

Entender as diferentes formas de interação entre os seres humanos e os animais dos ecossistemas no qual estão inseridos é de suma importância para a implantação de estratégias de conservação de fauna. Desse modo, com a obtenção de dados etnozoológicos, que permitem maior entendimento sobre o uso dos animais pela população estudada (PINTO, 2011; LIMA et al., 2018), é possível um melhor direcionamento para a elaboração de futuros trabalhos relacionados à conservação. Segundo Medeiros et al. (2011), se faz necessária a implantação de estratégias que promovam a sensibilização ambiental para que haja uma mudança de comportamento da população com relação ao uso dos recursos naturais.

As exposições itinerantes são um dos melhores métodos para divulgação de representações sociais e promoção de interações e alfabetização científica, enquanto recursos de ensino informal, possibilitando ainda a obtenção de dados sobre a percepção de uma população referente à determinado assunto (DELICADO, 2008; SANTOS; SCHULZE; WACHELKE, 2005).

Com base nessa afirmação, foram desenvolvidas exposições itinerantes com amostras de materiais relacionados aos quelônios amazônicos ocorrentes no Alto Juruá para serem aplicadas na Expo Acre Juruá, evento com repercussão regional com grande número de visitantes, que acontece no interior do Estado do Acre. Essa exposição teve por objetivo mostrar a importância da conservação das espécies de quelônios no ambiente, promovendo conhecimentos e sensibilização ambiental de forma dinâmica e de fácil compreensão ao público participante e obter dados sobre o consumo destes animais na região.

\section{Material e Métodos}

As exposições itinerantes de quelônios amazônicos foram desenvolvidas pela equipe do Projeto Bichos de Casco, vinculado ao laboratório de Biologia Animal da Universidade Federal do Acre (UFAC) - Campus Floresta e ocorreram durante a Expo Acre Juruá 2017, um evento regional com duração de quatro dias e teve como público médio de 20 mil pessoas por dia (SEBRAE). Durante esse período, foram realizadas amostras de banners informativos, algumas peças anatômicas de quelônios, animais fixados (Figura 1) e espécimes vivos de Chelonoidis denticulatus (Jabuti). Esses materiais pertencem ao laboratório de Biologia Animal da Universidade Federal do Acre (UFAC) - Campus Floresta. A exposição abordou temas como a conservação dos quelônios, promovendo informações sobre as espécies encontradas na Região Amazônica e os impactos causados na população em função de ações humanas.
Figura 1 - Exposição das peças anatômicas de quelônios e abordagem aos visitantes sobre o impacto antrópico e métodos de conservação

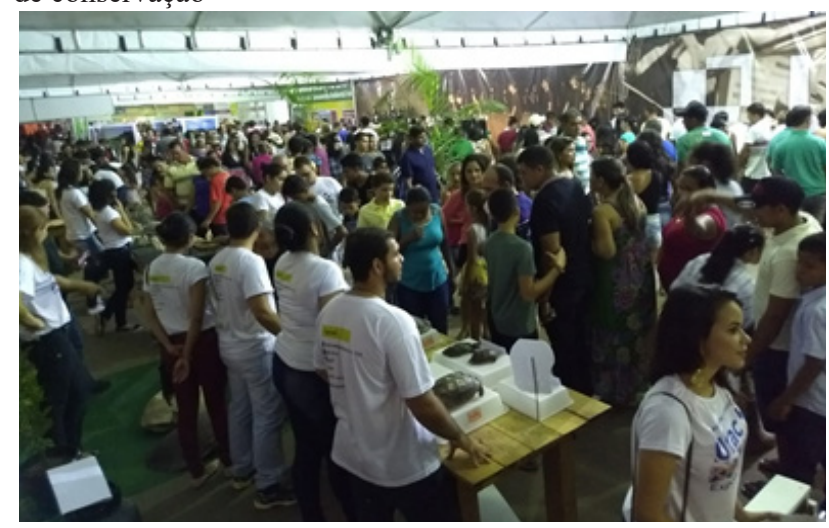

Fonte: Os autores.

Durante as exposições foram aplicados questionários semiestruturados (ALVES; SOUTO, 2011) aos visitantes que aceitaram participar voluntariamente da pesquisa. Entre as perguntas estavam questões relacionadas ao sexo, idade, consumo das espécies e conhecimentos relacionados ao aumento ou diminuição populacional do grupo. Essa pesquisa teve o intuito de adquirir dados sobre o consumo, comércio ilegal, domesticação, formas de captura e motivos pelos quais esses animais são utilizados como recurso alimentar.

\section{Resultados e Discussão}

Foram entrevistados homens e mulheres, na faixa etária de 16 a 58 anos. Com relação ao sexo dos entrevistados, houve uma diferença entre o número de homens e mulheres (Quadro 1), entretanto, essa variável não influencia nos dados com relação ao consumo em si, pois a maioria dos indivíduos que utiliza quelônios na alimentação são do sexo masculino (ATAÍDES et al.,2007; BERNHARD et al.,2016).

Quadro 1 - Número de entrevistados, por intervalos de idade e sexo

\begin{tabular}{|c|c|c|c|c|c|c|}
\hline \multirow{2}{*}{ Idade } & \multicolumn{2}{|c|}{ Masculino } & \multicolumn{2}{c|}{ Feminino } & \multicolumn{2}{c|}{ Total } \\
\cline { 2 - 7 } & $\mathbf{N}^{\mathbf{o}}$ & $\mathbf{( \% )}$ & $\mathbf{N}^{\mathbf{o}}$ & $\mathbf{( \% )}$ & $\mathbf{N}^{\mathbf{}}$ & $\mathbf{( \% )}$ \\
\hline $16-24$ & 3 & 15 & 3 & 15 & 6 & 30 \\
\hline $25-32$ & 3 & 15 & 0 & 0 & 3 & 15 \\
\hline $33-41$ & 4 & 20 & 1 & 5 & 5 & 25 \\
\hline $42-49$ & 1 & 5 & 1 & 5 & 2 & 10 \\
\hline $50-58$ & 3 & 15 & 1 & 5 & 4 & 20 \\
\hline Total & 14 & 70 & 6 & 30 & 20 & 100 \\
\hline
\end{tabular}

Fonte: Dados da pesquisa.

Com relação ao consumo de quelônios, 50\% $(n=10)$ dos entrevistados afirmaram utilizar desses animais na alimentação. Entre as espécies mais consumidas estavam o Jabuti $(C$. denticulatus), Tracajá (Podocnemis unifilis) e a Tartaruga da Amazônia ( $P$. expansa), conforme percentual de consumo apresentado no gráfico (Figura 2). A indicação de predileção pela espécie $C$. denticulatus difere da literatura, uma vez que as duas espécies do gênero Podocnemis foram relatadas como as de maior preferência alimentar para as populações 
tradicionais na Região Amazônica brasileira (ATAÍDES et al., 2007; BARROSO; MOURA, 2016; DE LA OSSA et al., 2011). Outras espécies citadas foram Iaçá (P. sextuberculata), Jurará (Platemys platycephala), Muçuã (Kinosternon scorpioides), Tartaruga de Igapó (Mesoclemmys gibba), Mata-matá (Chelus fimbriatus) e o Cabeçudo (Peltocephalus dumerilianus) (Figura 2). Entre as espécies menos consumidas estavam o Mata-matá e a Tartaruga de Igapó, cujo baixo índice de consumo está comumente associado com a dificuldade de encontros em seu ambiente natural, sua aparência e odores almiscarados. Tais características são relatadas como motivo de repulsa, reduzindo o consumo (SILVA et al., 2018).

Figura 2 - Porcentagem de consumo das espécies citadas no questionário.

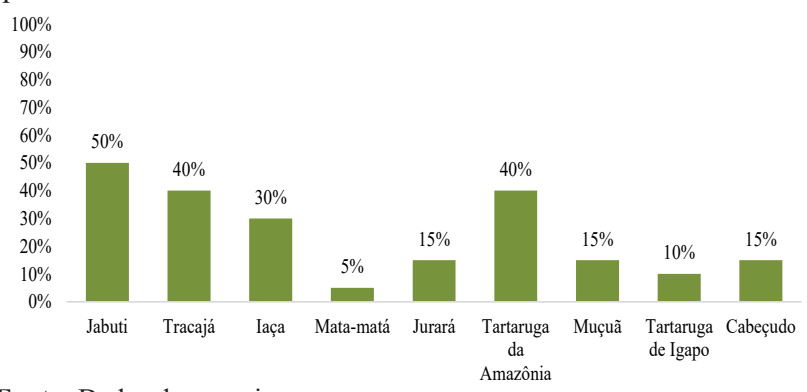

Fonte: Dados da pesquisa.

A captura de quelônios no ambiente natural é uma prática muito comum na Amazônia, tal como evidenciado por Brito et al. (2016). Apenas 40\% dos entrevistados relataram que quando encontram esses animais não os capturam, enquanto os outros $60 \%$ afirmaram capturá-los, seja para consumo direto (30\%), criação de estimação (15\%) ou engorda, alimentandoos até que o animal esteja maior e pronto para ser consumido, o período de engorda desses animais serve também para que deixe de ser "reimoso", (que faz mal a doentes) (15\%) tal como pode ser observado no Figura 3.

Figura 3 - Destinação dada aos animais encontrados ocasionalmente (dados em porcentagem)

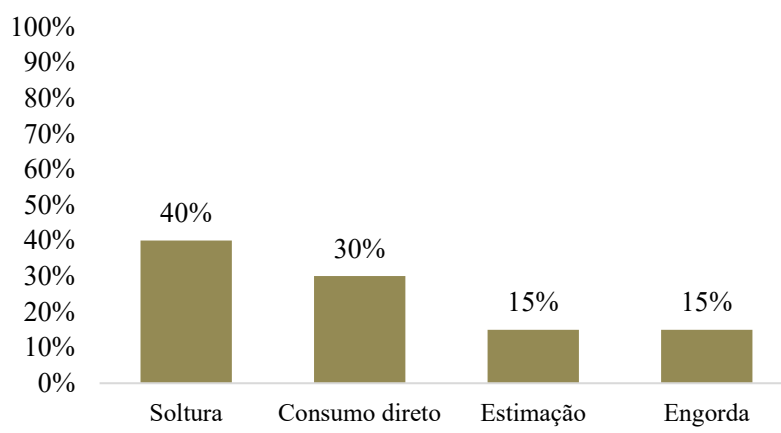

Fonte: Dados da pesquisa.

De acordo com Scarlato e Gaspar (2007), a criação de quelônios em cativeiro é frequente e está relacionada ao consumo de carne. Os entrevistados relataram uma grande diversidade de locais de destinação após a captura, sendo esses: Açudes (20\%), Cercados (30\%), Lagos (15\%), Banheiros (5\%), Bacias (10\%) e outros $(5 \%)$, enquanto $30 \%$ não capturam esses animais (Figura 4). O fato de a maioria dos animais ser colocados em Açude e Cercado se deve à preferência de captura de quelônios de grande porte, que não podem ser criados dentro de casa.

Figura 4 - Locais de contenção e criação dos animais capturados

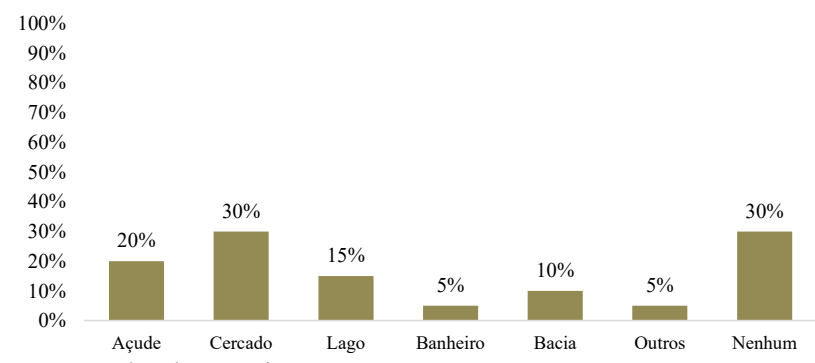

Fonte: Dados da pesquisa.

Quando questionados se houve mudança em relação à quantidade de quelônios encontrados na natureza ao longo do tempo, a grande maioria dos entrevistados relatou que ocorreu uma diminuição (70\%), 15\% relataram que não houve mudança, para $10 \%$ dos entrevistados houve aumento e $5 \%$ dos entrevistados responderam que não notaram aumento ou diminuição. O declínio populacional de quelônios no ambiente natural se deve, principalmente, ao comércio ilegal que vem sendo realizado há muitas décadas, com captura exacerbada de espécimes, sendo realizada muitas vezes no período reprodutivo dos animais (RÊBELO; PEZZUTI, 2000; EISEMBERG; VOGT, 2015), refletindo a necessidade de ações de conservação do grupo na região. Os quelônios apresentam baixa taxa de reposição de indivíduos na população, pois possuem lentas taxas de crescimento e longos períodos para alcançarem a maturidade sexual, características que já indicam a maior vulnerabilidade dessas espécies (POUGH; JANIS; HEISER, 2005).

Com relação ao tamanho dos animais, os mesmos 70\% que relataram que ocorreu uma diminuição do número da natureza, afirmaram que também houve uma redução no tamanho dos animais, $15 \%$ responderam que não perceberam mudança alguma, enquanto outros 15\% disseram desconhecer se houve aumento ou redução. A redução do tamanho desses animais se deve, principalmente, à exploração desenfreada dos indivíduos de maior tamanho (Silva et al., 2018).

Além do uso de quelônios para fins alimentares e como animais de estimação, estes animais são utilizados também na medicina tradicional (MARTINS; MOLINA, 2008; RUAS et al.,2017). No entanto, $70 \%$ dos entrevistados relataram não acreditar que esses animais possam ser utilizados para fins medicinais, enquanto os outros $30 \%$ afirmaram acreditar no uso desses animais para estes fins. Entre os usos dos quelônios para fins medicinais relatados pelos entrevistados estavam: uso da gordura do animal, como cicatrizante, cabeça de jabuti para tratar asma e para o aumento da possibilidade de se ter um filho homem, o uso do casco queimado para dores nas costas e, também, a cura da asma, usos semelhantes desses animais na medicina tradicional foram encontrados por 


\section{Oliveira et al.,(2019).}

Quando questionados se havia venda de quelônios no município de Cruzeiro do Sul - Acre, 40\% dos entrevistados afirmaram não saber, $35 \%$ relataram que não existe a venda e somente $25 \%$ afirmaram que os animais são vendidos na cidade. Entretanto, durante as entrevistas se notou que a maioria das pessoas tinha receio de responder essa pergunta, o que mostra que a maioria dos entrevistados estava devidamente informada que a venda desses animais no perímetro urbano se configura como tráfico, Lei ${ }^{\circ}$ 9.605/98 (BRASIL, 1998). Entre as espécies vendidas estavam o Jabuti (45\%), o Tracajá (10\%) e a Tartaruga da Amazônia (10\%) e $50 \%$ dos entrevistados não souberam informar.

\section{Conclusão}

As exposições itinerantes, realizadas na ExpoAcre Juruá, possibilitaram ao público presente interação e vivência em atividades com temáticas ambientais, promovendo conhecimentos e sensibilização para importância da conservação dos quelônios amazônicos. As entrevistas possibilitaram a obtenção de dados satisfatórios em relação à utilização e percepção do consumo de quelônios por moradores do município de Cruzeiro do Sul - Acre, que podem subsidiar ações de conservação. Com os resultados se pode concluir que exposições científicas são de grande valia para popularização da Ciência e os dados obtidos evidenciam a necessidade da realização de futuros projetos voltados para a conservação desses animais, envolvendo comunidades de perímetros urbanos.

\section{Referências}

ATAÍDES, A.G.; MALVASIO, A.; PARENTE, T.G. Percepções sobre o consumo de quelônios no entorno do Parque Nacional do Araguaia, Tocantins: conhecimentos para conservação. Gaia Scie., v.4, n.1, p.7-20, 2007.

BARRoso, W. A.; MOURA, N. A. Etnoconhecimento morfológico e ecológico de quelônios (Podocnemis expansa e $P$. unifilis) em uma comunidade ribeirinha. Biota Am., v.6, n.1, p.9195, 2016. doi: 10.18561/2179-5746/biotaamazonia.v6n1p91-95

BERNHARD, R. et al. Monitoramento da nidificação e manejo de ovos de quelônios amazônicos. In: FAGUNDES, C.K.; FERRARA, C.; BERNARDES, V.C.D. Manejo Conservacionista e Monitoramento Populacional de Quelônios Amazônicos. Brasília: IBAMA, 2016. p.40-51.

BRASIL. LEI No 9.605, DE 12 DE FEVEREIRO DE 1998. Dispõe sobre as sanções penais e administrativas derivadas de condutas e atividades lesivas ao meio ambiente, e dá outras providências. 1998.

BRITO, T.P.; LIMA, E.B.S.; ROSA, J.C.G.S. Avaliação do consumo de quelônios no município de Castanhal - Pará - Brasil. Rev. Ouricuri, v.6, n.1, p.71-103, 2016.

DE LA OSSA V.J.; OLIVERO-GOMEZ, G.; RUIZ, J.G. Utilización de quelonios de interés económico en el municipio de Caimito, Sucre, Colombia. Rev. Colomb. Cienc. Anim., v.3, n.1,

\section{p.3-14, 2011. doi: 10.24188/recia.v3.n1.2011.245}

DELICADO, A. Microscópios, batas brancas e tubos de ensaio: representações da ciência nas exposições científicas. Rev. Crít. Ciênc. Soc., v. 83, p.79-98, 2008. doi: 10.4000/rccs.454

EISEMBERG, C.C.; VOGT, R.C.. Vulnerabilidade dos ninhos de três espécies do gênero Podocnemis às mudanças climáticas em áreas protegidas da Amazônia. Relat. Conselho Nac. e Desenv. Cient. Tecnol., p. 37, 2015. doi: 10.13140/RG.2.2.32274.86725

FACHIN-TERÁN, A. Participação comunitária na preservação de praias para reprodução de quelônios na Reserva de Desenvolvimento Sustentável Mamirauá, Amazonas. Uacari, v.1, n.1, p.19-30, 2005. doi: 10.31420/uakari.v1i1.3

LIMA, J. S. et al. Etnozoologia e educação ambiental como ferramenta para a conservação dos animais. Rev. Ext. Uneal, v.4, n.2, p.9-16, 2018.

MARTINS, M.; MOLINA, F. B. Panorama geral dos répteis ameaçados do Brasil. In: ACHADO, A.; DRUMMOND, G.M.; PAGLIA, A.P. (Org.). Livro vermelho da fauna brasileira ameaçada de extinção. Brasília: Ministério do Meio Ambiente, 2008. p. 327-334.

MEDEIROS, A.B. et al. A Importância da educação ambiental na escola nas séries iniciais. Rev. Fac. Montes Belos, v.4, n.1, p.1-17, 2011.

OLIVEIRA, M.N et al. Impacto do consumo de quelônios no perímetro urbano de Cruzeiro do Sul - Acre - Brasil. Gaia Scie., v. 13, n. $4,2019$.

OLIVEIRA, P.H. de et al. Envolvimento comunitário na conservação de quelônios amazônicos. In: Manejo conservacionista e monitoramento populacional de quelônios amazônicos. Brasília: Ibama, 2016. p.29-34.

PINTO, L.C.L. Etnozoologia e conservação da biodiversidade em comunidades rurais da Serra do Ouro Branco. Biomas. Ufop. $\mathrm{Br}, 2011$.

POUGH, F.H.; JANIS, C.M.; HEISER, J.B. Vertebrate life. São Paulo: Pearson Prentice Hall, 2005.

REBÊLO, G.; PEZZUTI, J. Percepções sobre o consumo de quelônios na Amazônia: sustentabilidade e alternativas ao manejo atual. Amb. Soc., n.6/7, p.85-104, 2000. doi: 10.1590/S1414753X2000000100005.

RUAS, R.M.S. et al. Caça, captura e uso da fauna silvestre no brasil como crimes ambientais e tabu científico: reflexão sobre categorias teóricas. Holos, v.5, p.29, 2017.

SANTOS, M.E.; SCHULZE, C.M.N.; WACHELKE, J.F.R. A exposição itinerante enquanto promotora de divulgação científica: atitudes, padrões de interação, e percepções dos visitantes. Psicol. Teoria Prática, v.7, n.2, p.49-86, 2005.

SCARLATO, R.C.; GASPAR, A. Composição nutricional do casco da tartaruga-da-Amazônia (Podocnemis expansa) criada em cativeiro e em idade de abate. Food Scie. Technol., v.27, n.suppl 1, p.41-44, 2007. doi: 10.1590/S0101-20612007000500008

SILVA, A.L.; BEGOSSI, A. Uso de recursos por populações ribeirinhas do médio Rio Negro. In: BEGOSSI, A. (Ed). Ecologia de pescadores da Mata Atlântica e da Amazônia. São Paulo: RIMA, 2003. p.87-145.

SILVA, T.L. et al. Perspectivas de estudantes sobre a conservação de quelônios em uma escola da zona periférica de Cruzeiro do Sul - Acre. Rev. Communitas, v.2, n.3, p.304-313, 2018. 